\title{
Editorial
}

\section{No benefit treating mild hypertension}

Journal of the Ceylon College of Physicians, 2012, 43, 1

The 2012 Cochrane Review on "Pharmacotherapy for Mild Hypertension" concluded that treating patients with stage 1 (mild) hypertension has no benefit, from studies conducted in the United Kingdom, Australia, and the United States. Data from four randomised controlled trials, involving 8912 patients with stage 1 hypertension (systolic blood pressure 140-159 mm Hg or diastolic $90-99 \mathrm{~mm} \mathrm{Hg}$, or both) and treated for four to five years, found that drug treatment did not reduce total mortality (risk ratio 0.85 (95\% confidence interval 0.63 to1.15), coronary heart disease (1.12 (0.8 to 1.57)), or stroke $(0.51(0.24$ to 1.08$)$. Patients with preexisting cardiovascular disease were excluded from the study.

This means that millions are being overtreated, but the guidelines (NICE) recommend treating mild hypertension only after a risk assessment using the QRisk algorithm to predict cardiovascular disease. But as we know many doctors ignore this advice and treat according to BP readings. It is much easier to treat with a drug rather than to explain to a patient that he need not take a drug due to his very low risk and that he needs only life style modification. Also "what if?" he gets a stroke or MI despite the low risk? What will the patient and the family think of you? How will you explain to them the correctness of your decision?

Redefining hypertension threshold from a previous $160 / 100$ thirty years ago to 2003 ESC cutoff of 140/90 would create 13 million hypertensive patients in United States alone. Every clinician knows the fear aroused in many patients by a diagnosis of hypertension and this fear has tangible outcomes such as work absenteeism and family stress. In the Cochrane review around $9 \%$ of patients with mild hypertension have stopped treatment due to adverse effects and they have suffered these adverse effects by taking a drug with no proven benefit for them. In view of the mounting evidence of both waste and harm, it would be prudent to consider changing rules: Going back to previous treatment thresholds of $160 / 100$ for the otherwise healthy people. However in the trials included in the Cochrane Review majority of the patients were taking only a beta blocker. Recent systematic reviews have shown that beta blockers do not provide the same mortality benefit as other newer antihypertensives. Also, the total number of events in the studies was small; suggesting that a large well designed study comparing ACE inhibitors/blockers with placebo in mild hypertensives is needed.

\section{Saman Gunatilake Co-Editor}

